\title{
Standards Proposal for 2021 to Support Planetary Coordinate Reference Systems
}

\author{
Trent M. Hare ${ }^{\mathrm{a}, ~ *}$, Jean-Christophe Malapert ${ }^{\mathrm{b}}$ \\ ${ }^{a}$ U.S. Geological Survey, Astrogeology, Flagstaff, AZ, 86001, thare@usgs.gov \\ ${ }^{b}$ Centre National d'Etudes Spatiales (CNES), jean-christophe.malapert@cnes.fr \\ * Corresponding author
}

Keywords: Map projections, OGC, Web Services, Planetary

\begin{abstract}
:
This abstract outlines an updated proposal to encode planetary map projections for open geospatial consortium (OGC) web services. The update leverages the recent availability of the International Organization for Standardization (ISO) 19162 standard, also known as WKT2. ISO 19162 standard defines a well-known text (WKT) definition for coordinate reference systems (CRS) and has been improved to support nearly all planetary CRS needs. These codes are needed to allow streaming mapping applications to recognize a defined planetary CRS and give them the capability to overlay multiple data sets from different sources and implement accurate measures and calculations.
\end{abstract}

Hare et al. in 2006 originally proposed methods to support planetary CRSs within existing OGC web mapping standards. For a typical OGC web mapping session, the server must define a minimum set of information such that the client application understands not only the data layer but also the current CRS and/or map projection. Generally, web mapping servers only support the numeric European Petroleum Survey Group (EPSG) codes to define the CRS also referred to as the Spatial Reference System (SRS). For example, code "4326" is the EPSG identifier for Earth's "WGS 84" geographic CRS. To help solve this incompatibility among planetary clients and servers, the authors have proposed their own set of codes outside of the EPSG namespace, but for eventual adoption by the OGC. The proposed codes will leverage both the Navigation and Information Facility numeric code for extraterrestrial bodies and the IAU's publication date from the Report on Cartographic Coordinates and Rotational Elements.

NAIF codes define the barycenter (center of mass) of the Solar System as 0 and defines the Sun as 10. This allows the planets to be classified as 1 through 9 starting with Mercury out to Pluto (even though Pluto is now considered only a dwarf planet). The NAIF planet ID is then defined as the planet barycenter ID *100+99. Thus Mars, in the NAIF system, is defined as " 499 ". To build upon that value for our proposed planetary IAU codes, the new geospatial code for Mars is derived as follows allowing for 100 codes for Mars including several predefined map projections.

- Mars IAU:2018 code $=499 * 100=49900$

- Sample WMS call: "SRS=IAU:2018:49900"

While the proposal to support EPSG-like planetary codes is not ideal, we feel it is still required to support exiting OGC web protocols. We also plan to include these codes within the PROJ library for use within applications like GDAL and QGIS. And finally, we will continue to push the more robust WTK v2 standard for file-based and forthcoming streaming standards and continue to work with the IAU Working Group on Cartographic Coordinates and Rotational Elements to inform and coordinate these efforts (https://github.com/PlanetMap/csvForWKT).

\begin{tabular}{|l|l|}
\hline \multicolumn{1}{|c|}{ IAU:xxx00 } & $\begin{array}{l}\text { Ocentric on a sphere. If triaxial use IAU Mean radius. If elliptical use semi-major axis as a sphere, (always east } \\
\text { longitude direction). This is to enable the most interoperable definition for the body. }\end{array}$ \\
\hline IAU:xxx01 & Ographic on ellipse, (east or west, depending on body rotation). \\
\hline IAU:xxx02 & Ocentric on ellipse, (always east). \\
\hline IAU:xxx03 & Ographic on triaxial, (east or west, depending on body rotation). \\
\hline IAU:xxx04 & $\begin{array}{l}\text { Ocentric on triaxial, (always east). Note triaxial definitions are not generally supported in typical mapping } \\
\text { applications but may be useful for more advanced applications like ISIS, VICAR, etc. }\end{array}$ \\
\hline $\begin{array}{l}\text { IAU:xxx05 } \\
\text { xxx9 }\end{array}$ & $\begin{array}{l}\text { Reserved for special cases uniquely defined per body. For example, Mars 49905 can be used for the "truncated" } \\
\text { Mars radius 3396000 (m) as defined by some instrument teams (e.g., MOLA and HRSC Team). In rare cases a } \\
\text { reserved code may be defined to support using a polar radius as a sphere. }\end{array}$ \\
\hline
\end{tabular}

Table 1: List of the five CRS options per body in degrees using the defined IAU radius value(s) \& longitude direction. 